Genetics Service, Royal Children's Hospital, Parkville, Melbourne, Australia

Comprehensive genomic profiling (CGP) describes technologies that allow profiling of all major types of genomic alterations, including simple nucleotide variation (SNV and indel), somatic copy number alteration (SCNA), structural variation $(\mathrm{SV})$, and aggregate markers of genome damage such as tumour mutation burden (TMB) and microsatellite instability (MSI). CGP could lead to improved access to molecularly targeted therapies for cancer patients but, aside from a few accredited commercial and academic providers, is not widely used in routine pathology practice. Aspects of CGP presenting barriers to broad adoption include cost, technical complexity, lack of reimbursement, and a perceived lack of clinical utility. Here we summarise the experience of Peter Mac's Pathology Department in providing CGP in the translation research setting over three years of testing for personalised medicine trials. We find that characterising tumours using a comprehensive in-house clinical research panel provides useful diagnostic and predictive information that impacts patient care in a number of cases, however at relatively high per sample cost. In comparison, we describe our experience with one of the first kit-based CGP research products on the Australian market meeting the requirements for routine CGP.

\section{IMPROVING GENOMICS AND BIOINFORMATICS LITERACY IN THE UNDERGRADUATE MEDICAL SCIENCE CURRICULUM}

\author{
Christine Knauth $^{1,2}$ \\ ${ }^{1}$ School of Biomedical Sciences, Queensland University of \\ Technology, Brisbane, Qld, Australia; and ${ }^{2}$ Australian Red \\ Cross Blood Service Research and Development, Australian \\ Red Cross Lifeblood, Kelvin Grove, Qld, Australia
}

Background: The growth of genetic sequencing requests for pathology diagnoses and personalised medicine demands that graduating medical scientists be cognisant of genomics and bioinformatics, in preparation for their future careers.

Aim: To improve the genomic and bioinformatics literacy of undergraduate Medical Science students.

Methods: QUT Bachelor of Medical Science students enrolled in a fourth-year core unit, studied a series of lectures and workshops covering genomic and bioinformatics analysis of bacterial, human germline and somatic case studies relevant to pathology diagnosis. The bioinformatics platform used was Galaxy. Students voluntarily and anonymously completed a survey prior and subsequent to the workshops to ascertain their growth in genomic literacy.

Results: Fifty-five students completed the pre-workshops survey: eight students declared 'very little' prior experience with bioinformatics, and four declared some experience in coding. The other students declared no previous experience. Fifteen students completed the post-workshop survey, of which 14 were 'a bit confident' or 'moderately confident' in their abilities. Eight students were moderately or strongly interested in continuing study in this area.

Discussion: Embedding and advancing teaching and learning of clinical genomics and bioinformatics in the undergraduate Medical Science curriculum is crucial for providing career-ready graduates in this burgeoning discipline of pathology.

\section{URINE ORGANIC ACID (UOA) ANALYSIS FOR THE DIAGNOSIS OF AROMATIC L-AMINO ACID DECARBOXYLASE (AADC) DEFICIENCY}

\section{Chun Yiu Law ${ }^{1}$, Tsz Ki Ling ${ }^{1}$, Ka Chung Wong ${ }^{1}$,} Ching Wan Lam ${ }^{1,2}$

${ }^{1}$ Division of Chemical Pathology, Department of Pathology, Queen Mary Hospital, Hong Kong, China; and ${ }^{2}$ Department of Pathology, University of Hong Kong, Hong Kong, China

We describe the use of urine organic acid (UOA) analysis for the diagnosis of a CSF neurotransmitter defect, aromatic L-amino acid decarboxylase (AADC) deficiency in a 3-month-old boy. The patient presented with upper limbs spasm and abnormal eye movement. UOA was initiated for possible inborn errors of metabolism (IEM). Hyper-excretion of urine vanillactic acid (VLA) and $\mathrm{N}$-acetyl-vanilalanine was detected in UOA which the pattern is pathognomonic to AADC deficiency. The diagnosis was subsequently confirmed by analysing the hotspot pathogenic variant in the $D D C$ gene, i.e., IVS6+4A $>\mathrm{T}$ in $<2$ days. A CSF neurotransmitter profile was requested by the clinician and showed a classical pattern of AADC deficiency with markedly elevated 3-O-methyldopa of $2895 \mathrm{nmol} / \mathrm{L}(\mathrm{RI}<300)$ and very low levels of 5-HIAA ( $<17 \mathrm{nmol} / \mathrm{L} ;$ RI 114-335) and HVA $(<67$ nmol/L; RI 295-932). Because CSF sampling can be technically challenging and is also invasive, our case illustrates the potential of using non-invasive UOA to replace CSF neurotransmitter for the diagnosis of AADC. This is particularly useful clinically if sampling of CSF is practically infeasible. In conclusion, UOA is a simple and non-invasive test for AADC deficiency, and substantiate the previous work reported by Lee et al. ${ }^{1}$

Reference

1. Lee HC, Lai CK, Yau KC, et al. Non-invasive urinary screening for aromatic L-amino acid decarboxylase deficiency in high-prevalence areas: a pilot study. Clin Chim Acta 2012; 413: 126-30.

\section{IMPACT OF MATERNAL EXERCISE ON THE FRACTION OF FETAL CELL FREE DNA IN MATERNAL PLASMA}

Abbey Clarence ${ }^{1}$, Gustaaf Dekker ${ }^{2,3}$, Margaret Arstall ${ }^{3,4}$, Eric Lee $^{5}$, Stephanie Grehan ${ }^{5}$, Aimee Jordan ${ }^{5}$, Jill Hall ${ }^{1}$, James Harraway, Graeme Suthers ${ }^{1,6}$

${ }^{1}$ Clinpath Laboratories, SA, Australia; ${ }^{2}$ Department of Obstetrics, Lyell McEwin Hospital, Northern Adelaide Local Health Network, SA, Australia; ${ }^{3}$ University of Adelaide, SA, Australia; ${ }^{4}$ Department of Cardiology, Lyell McEwin Hospital, Northern Adelaide Local Health Network, SA, Australia; ${ }^{5}$ Sullivan Nicolaides Pathology, Qld, Australia; and ${ }^{6}$ Sonic Healthcare (Australia), NSW, Australia

\footnotetext{
Aim: Maternal exercise prior to non-invasive prenatal testing (NIPT) may reduce the fraction of cell free DNA attributable to the fetus ('fetal fraction'), a key determinant of NIPT performance. This pilot study aimed to assess the impact of maternal exercise on fetal fraction.

Methods: 15 pregnant women of gestational age $\geq 10$ weeks were recruited from a public hospital antenatal service. Moderate exercise was undertaken (clinical exercise stress test as per Bruce protocol with target heart rate of $140 \mathrm{bpm}$ ), with peripheral blood collected pre- and post-exercise. Gestational age, maternal age, maternal body mass index (BMI), heart rate, and blood pressure were recorded. Fetal fraction was determined by the Harmony NIPT assay.
} 
Results: As a cohort, there was no significant difference in postexercise fetal fraction $(p=0.38)$. However, women with BMI $<25$ had an average fetal fraction change of $-2.3 \%(n=5, p=0.02)$, while women with BMI $\geq 25$ had an average change of $+0.5 \%$ $(n=10, p=0.25)$. Change in fetal fraction was not influenced by gestational age, maternal age, change in heart rate, or change in systolic blood pressure.

Discussion: Moderate maternal exercise may only impact fetal fraction when maternal BMI is $<25$. Further studies are required to confirm this association.

\section{MEASURABLE RESIDUAL DISEASE (MRD) DETECTION BY NEXT GENERATION SEQUENCING (NGS) IN ACUTE LYMPHOBLASTIC LEUKAEMIA (ALL)}

Wendi Lin $^{1}$, Rishu Agarwal ${ }^{2}$, Suzanne Svobodova ${ }^{2}$, Chun Yew Fong

${ }^{1}$ Clinical Haematology, Austin Health, Melbourne, Australia; and ${ }^{2}$ Molecular Diagnostics, Austin Pathology, Melbourne,

Australia

Introduction: MRD, a key prognostic factor in ALL, is traditionally assessed by flow cytometry and/or allele specific oligonucleotide PCR (ASO-PCR). Here we describe validation and implementation of novel NGS technologies to measure MRD at clinically relevant time points.

Methods: Extracted DNA from bone marrow were analysed using the LymphoTrack ${ }^{\circledR}$ Diagnostic Assay Panel to detect $\operatorname{IgH}$ gene rearrangements. Sequence clonality was defined as $>2.5 \%$ of total reads and $>2 \mathrm{x}$ frequency of the third most frequent sequence. A 100 cell equivalent spike-in control (LymphoQuant ${ }^{\mathrm{TM}}$ ) was utilised for normalisation.

Results: Results were concordant $(78.18 \%)$ between assay methods with the exception of 12 samples. In 10 cases, MRD was detected by NGS at a lower level than flow cytometry and ASOPCR, which were negative. MRD positivity by NGS corresponded with poor clinical outcomes in these patients. We demonstrate that the use of spike-in controls deliver clinically meaningful results. Conclusion: MRD detection by NGS is complementary to testing using flow cytometry and ASO-PCR. NGS has the potential advantage of increased sensitivity, detection of clonal evolution and a rapid turnaround time. Normalisation of MRD levels to cell equivalents is required to suitably compare results with flow cytometry and ASO-PCR.

\section{REVIEW OF 12 MONTHS OF COPY NUMBER VARIANT CALLING ON A CLINICAL NEXT GENERATION SEQUENCING PIPELINE}

Dylan A. Mordaunt ${ }^{1,2}$, Julien Soubrier ${ }^{1}$, Song Gao ${ }^{1}$, Lesley Rawlings $^{1}$, Jillian Nicholl ${ }^{1}$, Sui $\mathrm{Yu}^{1}$, Janice Fletcher ${ }^{1}$, Karin Kassahn

${ }^{1}$ Genetics and Molecular Pathology, SA Pathology, SA, Australia; and ${ }^{2}$ Department of Paediatrics, School of Medicine, University of Adelaide, SA, Australia

Background: Next generation sequencing (NGS) provides the opportunity to detect not only single nucleotide variants and small indels, but also larger whole exon or whole gene deletions and duplications (CNVs).
Method: We implemented CNV calling in our NGS pipeline utilising the DECoN, XHMM and PanelCNV packages. We compared the performance with multiplex ligation-dependent probe amplification (MLPA) on our 1200 gene panel (R1kD) and with chromosomal microarray (CMA) on our whole-exome (WES) platform.

Results: On the R1kD, we reviewed 197 patient cases which had both NGS and MLPA. We detected 25 deletions and 7 duplications, all of which were detected by both methods. Seven other CNV calls from NGS were considered artefacts during IGV review due to the presence of pseudogenes and poor mapping. On the WES platform, we reviewed 37 patient cases which had both NGS and CMA and detected 11 deletions and 10 duplications by both methods. NGS called some additional smaller events which were not reported by CMA.

Conclusions: CNV analysis on NGS can be implemented in clinical pipelines. Based on review of the first 12 months of service we have moved some tests to NGS with MLPA only performed to check positive results.

\section{HEPARIN INDUCED THROMBOCYTOPENIA IN PATIENTS WITH SEVERE RHABDOMYOLYSIS: A CASE SERIES}

Roya Arabi ${ }^{1,2}$, Jessica Margaret Heenan ${ }^{1}$, Julia Helen Gardner ${ }^{1}$, Muhajir Mohamed ${ }^{1,3}$

${ }^{1}$ Launceston General Hospital, Launceston, Tas, Australia;

${ }^{2}$ Royal Hobart Hospital, Hobart, Tas, Australia; and

${ }^{3}$ University of Tasmania, Launceston, Tas, Australia

Heparin-induced thrombocytopenia (HIT) is a known immunological complication of heparin administration which can cause life threatening thrombosis. Here, we report a series of three cases of HIT in the context of acute kidney injury due to rhabdomyolysis that presented to Launceston General Hospital, Tasmania, between December 2017 and December 2018. During this time, there were a total number of four patients with HIT, out of which three had rhabdomyolysis. Patients were all men with age between 29 and 68 years old. The causes of rhabdomyolysis were statin therapy (in two cases) and ischemia induced by compartment syndrome (in one case). All patients received unfractionated heparin and continuous renal replacement therapy for 2-11 days. 4Tscore was 4 in one patient and 5 in two other patients. None of patients had thrombosis complication. In all patients, anti-heparin/PF4 antibody was detected by BioRad Heparin/PF4 gel column assay and the diagnosis was confirmed by serotonin release assay. Patients received non-heparin anticoagulation. Platelet count recovered after cessation of heparin. Since rhabdomyolysis is an uncommon condition, the concomitant development of HIT in our 3 cases raises the suspicion whether administration of unfractionated heparin in patients with rhabdomyolysis poses an additional risk for developing HIT.

\section{IDARUCIZUMAB RESISTANCE DUE TO A MARKEDLY ELEVATED DABIGATRAN CONCENTRATION - A CASE REPORT}

\footnotetext{
H. Cashman ${ }^{1,2}$, J. Joseph ${ }^{1,2}$, D. Roberts ${ }^{1,2,3}$

${ }^{1}$ Department of Haematology, St Vincent's Hospital, Sydney, Australia; ${ }^{2}$ University of New South Wales, Sydney, Australia;
} 